\title{
PREVALÊNCIA E IMPACTO DA MIGRÂNEA EM FUNCIONÁRIOS DO HOSPITAL DAS CLÍNICAS DA FACULDADE DE MEDICINA DE RIBEIRÃO PRETO - USP
}

\author{
MARCELO EDUARDO BIGAL *, LUCIANA CAMPANER FERNANDES **, FÁBIO A. MORAES ***, \\ CARLOS ALBERTO BORDINI****, JOSÉ GERALDO SPECIALI*****
}

\begin{abstract}
RESUMO - Cefaléia é sintoma comum na população, com prevalência ao longo da vida superior a $90 \%$. Acarreta importante impacto na qualidade de vida de seus portadores. O presente estudo tem por objetivo avaliar a prevalência da migrânea entre trabalhadores de um hospital universitário, bem como mensurar a intensidade, interferência e impacto dela em algumas atividades cotidianas. Um total de 1890 funcionários respondeu a questionário que possibilitava o diagnóstico da migrânea. A prevalência dessa cefaléia ao longo da vida foi $30,4 \%$. A dor foi considerada como intensa, na maioria das vezes, por $86 \%$ dos migranosos. Verificou-se impacto importante em aspectos da vida cotidiana tanto durante como no intervalo das crises de dor. Conclui-se que a migrânea representa problema de saúde pública entre os funcionários do HC. Por acarretar importante impacto na qualidade de vida desses trabalhadores, é possível haver diminuição da capacidade laborativa deles, com prejuízo econômico considerável. Cremos que este problema mereça, portanto, atenção especial, através de melhor diagnóstico e tratamento.
\end{abstract}

PALAVRAS-CHAVE: migrânea, prevalência, qualidade de vida.

\section{Migraine prevalence and impact: an university hospital employees based study}

\begin{abstract}
Headache is a common symptom in the population, with a life prevalence around $90 \%$. It results in an important impact in the life quality of sufferers. The aim of this study is to evaluate the prevalence of migraine among employees of an university hospital $(\mathrm{HC})$, as well as to measure the headache intensity, interference and impact in the daily activities. A total of 1890 employees had answered to a questionnaire which made possible to carry out diagnosis of migraine. Life prevalence of this headache type was $30.4 \%$. Pain was considered intense, most of the time, by $86 \%$ of the migraneurs . It was verified an important impact in the daily life aspects as much during as between the headache attacks. It can be concluded that migraine represents a public health problem among the $\mathrm{HC}$ employees. Because migraine brings about an important impact in the life quality of those workers, it is possible that a reduction of working capacity with considerable economic burden exists. This problem deserves, thus, special attention, through a better diagnostic and treatment.
\end{abstract}

KEY WORDS: migraine, prevalence, life quality.

Desde os primórdios da civilização, o ser humano preocupa-se com a cefaléia. Referências sobre ela existem na literatura médica pré-hipocrática. A epidemiologia das cefaléias primárias tem sido estudada durante séculos, mas só nas últimas duas décadas houve progresso significativo no seu entendimento e sistematização. Sabe-se que a prevalência ao longo da vida, na população norte-

*Mestre em Neurologia pela Faculdade de Medicina de Ribeirão Preto (FMRP) da Universidade de São Paulo (USP); **Psicóloga, pós-graduanda em Neurociências da FMRP/USP; ***Médico Residente do Hospital das Clínicas (HC) da FMRP/USP; ****Doutor em Neurologia pela FMRP/USP; *****Professor Associado de Neurologia da FMRP/USP.

Dr. José G. Speciali - Faculdade de Medicina de Ribeirão Preto, Departamento de Neurologia - Av. Bandeirantes 3900 - 14049-900 Ribeirão Preto SP - Brasil. 
americana, encontra-se acima de $90 \%$. Além disso existem estudos demonstrando aumento de até $60 \%$ na prevalência atual cefaléia, frente a observada 10 anos atrás ${ }^{1-5}$.

Em relação à migrânea (enxaqueca), estima-se que pelo menos 3\% a 6\% dos homens e 13\% a $18 \%$ das mulheres a apresentem ${ }^{6-7}$. A qualidade de vida dos indivíduos acometidos está comprometida, por vários motivos ${ }^{8-11}: 1$ - Estas pessoas têm maior incidência de dores no corpo, limitação física e consideram-se com menor saúde mental. A enxaqueca prejudica mais a qualidade de vida que a osteoartrite, diabetes, hipertensão e lombalgia e é tão incapacitante quanto a depressão. 2 - Há comprometimento de suas vidas profissionais, devido a maior número de faltas e menor atividade laborativa secundárias à dor ou aos sintomas associados. O nível de ganho monetário dos enxaquecosos é menor que o da população geral. 3 - A vida social, familiar e escolar dos pacientes é comprometida. Esse fatores representam impacto físico, mental e social para os migranosos, bem como implicam em altos custos para a sociedade.

Apesar disso ainda são pouco frequentes os estudos epidemiológicos realizados em países em desenvolvimento. Nosso estudo tem por objetivo avaliar a prevalência da migrânea entre trabalhadores de um hospital universitário, bem como mensurar a intensidade, interferência e impacto em algumas atividades cotidianas de seus portadores.

\section{MÉTODO}

O presente estudo foi realizado no Hospital das Clínicas da Faculdade de Medicina de Ribeirão Preto USP (HC). Em um primeiro momento, 1890 funcionários, em presença de um entrevistador, responderam a um questionário (questionário padrão) que apresentava, em termos leigos, os critérios diagnósticos para migrânea da Sociedade Internacional de Cefaléia (SIC) ${ }^{12}$. Definiram-se então 4 grupos: 1 - funcionários sem cefaléia; 2 funcionários que apresentavam algum tipo de cefaléia que não preenchia critérios para migrânea; 3 - funcionários cuja dor preenchia todos os critérios para migrânea; 4 - funcionários cuja dor preenchia critérios para migrânea, porém durava menos que 4 horas. Avaliou-se a prevalência ao longo da vida, no último ano e no último mês. Considerou-se, para efeito de cálculo de prevalência anual, aqueles funcionários que apresentaram mais de 4 episódios de migrânea ao longo da vida e pelo menos 1 episódio no último ano. De maneira semelhante, para o cálculo de prevalência mensal. Os funcionários que enquadravam-se nos Grupos 3 e 4 (dor com características migranosas, mesmo que durassem menos que 4 horas) foram então procurados para que respondessem um segundo questionário, com perguntas acerca da intensidade e interferência da dor (questionário complementar).

No sentido de validar o questionário padrão, 360 funcionários com possível migrânea (Grupos 3 e 4) foram submetidos, quando da aplicação do questionário complementar, à consulta com um mesmo neurologista (MEB), havendo posterior análise da especificidade dele. Além disso, 50 funcionários com cefaléia que não preenchia os critérios para migrânea (Grupo 2), de acordo com o questionário padrão, também submeteram-se à consulta.

A intensidade e a interferência da dor foram aferidas através da aplicação do Índice de Dor Crônica, proposto por Von Korff et al. ${ }^{13}$ (índice de Von Korff) aplicado dentro do questionário complementar. Esse índice classifica uma dor crônica em categorias (Tabela 1).

O questionário complementar apresentava ainda perguntas sobre o impacto da cefaléia durante e entre as crises de dor. Tais questões estão apresentadas nas Tabelas 3 e 4.

Tabela 1. Índice de dor crônica ${ }^{13}$.

Índice de dor

0

1

2

3

4
Significado

Sem dor nos últimos 6 meses.

Baixa intensidade e baixa interferência

Alta intensidade e baixa interferência

Moderadamente limitante

Severamente limitante 
Tabela 2. Prevalência de migrânea e migrânea exceto um critério (duração $<4$ horas) entre funcionários do HC.

\begin{tabular}{llcc}
\hline & $\begin{array}{c}\text { Migrânea } \\
\text { grupo 3) } \\
\mathrm{n}(\%)\end{array}$ & $\begin{array}{c}\text { Migrânea com dor } \\
4 \text { horas }(\text { grupo 4) } \\
\mathrm{n}(\%)\end{array}$ & $\begin{array}{c}\text { Total } \\
(\text { grupos 3 e 4) } \\
\mathrm{n}(\%)\end{array}$ \\
\hline $\begin{array}{l}\text { Prevalência ao longo da vida } \\
\text { Prevalência anual }\end{array}$ & $575(30,4)$ & $271(14,3)$ & $846(44,7)$ \\
Prevalência mensal & $557(29,5)$ & $263(13,9)$ & $820(43,4)$ \\
\hline
\end{tabular}

n, valor absoluto; \%, valor relativo.

Tabela 3. Impacto da cefaléia sobre algumas atividades diárias.

\begin{tabular}{lcc}
\hline Durante a crise de dor você... & $\begin{array}{c}\text { Sim } \\
\mathrm{n}(\%)\end{array}$ & $\begin{array}{c}\text { Não } \\
\mathrm{n}(\%)\end{array}$ \\
\hline Tem medo de dirigir?* & $206(35,1)$ & $380(64,9)$ \\
Já cancelou atividades com a família? & $540(70)$ & $231(30)$ \\
Já desmarcou atividades de lazer? & $626(81,2)$ & $145(18,8)$ \\
Tem que se deitar?** & $193(25)$ & $139(18)$ \\
\hline
\end{tabular}

n, valor absoluto; \% valor relativo.

*Considerados apenas os funcionário que sabem dirigir.

**57\% dos funcionários referiram que gostariam de se deitar mas não podem por algum motivo.

Tabela 4. Avaliação do impacto causado pela enxaqueca entre as crises de dor.

\begin{tabular}{lccc}
\hline Entre as crises de dor...* & $\begin{array}{c}\text { Sim } \\
\mathrm{n}(\%)\end{array}$ & $\begin{array}{c}\text { Não } \\
\mathrm{n}(\%)\end{array}$ & $\begin{array}{c}\text { Não sei } \\
\mathrm{n}(\%)\end{array}$ \\
\hline ... Você fica sempre pensando na dor? & $361(46,8)$ & $309(40,1)$ & $101(13,1)$ \\
... Você tem muito medo de ter outra crise? & $331(42,9)$ & $373(48,4)$ & $67(8,7)$ \\
... Há prejuízo de seu relacionamento familiar? & $186(24,1)$ & $540(70)$ & $45(5,9)$ \\
... Há prejuízo de sua atividade sexual? & $139(18)$ & $495(64,2)$ & $137(17,8)$ \\
... Há prejuízo de seu relacionamento com \\
amigos ou colegas de serviço?
\end{tabular}

*Em todas as questões frisava-se bem que se tratava de situações entre as crises de dor.

\section{RESULTADOS}

Foram 1890 os funcionários que responderam ao questionário padrão. Desses, 664 (35,1\%) referiram não apresentar cefaléia, sendo 322 (48,5\%) masculinos e 342 (51,5\%) femininos.

Dos pacientes com cefaléia, 380 (20,1\%), apresentavam outros tipos que não migrânea (Grupo 2), 31 (1,6\%) apresentando cefaléia com características migranosas, porém frequência maior que 15 episódios por mês (cefaléia crônica diária, tipo migrânea transformada).

Assim, 846 funcionários apresentaram dor classificada nos Grupos 3 e 4, sendo 167 (19,8\%) masculinos e $679(80,2 \%)$ femininos. Trezentos e sessenta funcionários desses grupos foram submetidos a consulta neurológica, observando-se concordância diagnóstica em 339 (94,2\%), corroborando a especificidade do método. 


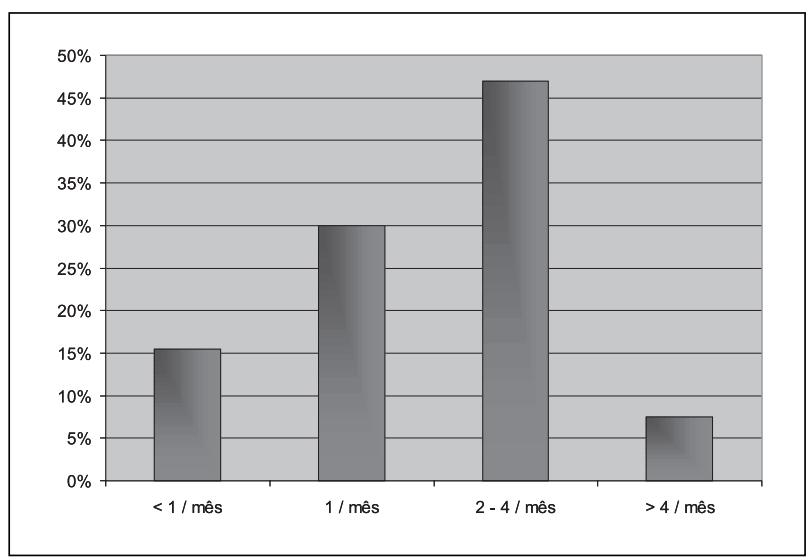

Fig 1. Frequência de crises migranosas entre funcionários do HC.

As prevalências encontradas são apresentadas na Tabela 2.

A frequência das crises, dentre os pacientes com pelo menos uma crise no último ano (820) está apresentada em gráfico (Fig 1), tendo sido considerados em conjunto os pacientes dos grupos 3 e 4 .

Dentre os 846 pacientes classificados nos Grupos 3 e 4, 771, todos tendo apresentado dor no último ano, foram submetidos ao questionário complementar. Os demais não foram localizados ou se recusaram a responder o questionário. A intensidade e interferência global da dor foi graduada através do índice de dor crônica e os resultados estão apresentados no gráfico (Fig 2).

O impacto que a crise de migrânea acarreta em aspectos da vida cotidiana do funcionário está apresentado na Tabela 3.

A Tabela 4 mostra o resultado de algumas perguntas formuladas com o sentido de avaliar o impacto da cefaléia entre as crises de dor.

\section{DISCUSSÃO}

Cefaléia é sintoma frequentemente encontrado na prática clínica. Rassmussen, na Dinamarca, encontrou prevalência ao longo da vida entre 93 e 96\% . Pesquisas realizadas no Brasil chegaram a resultados semelhantes ${ }^{14}$. No entanto, considera-se classicamente a prevalência da cefaléia do tipo tensional como sendo maior que a da migrânea. Em estudo de 1995, Rasmussen encontrou prevalência ao longo da vida de $15 \%$ para a migrânea e $69 \%$ para cefaléia do tipo tensional episódica ${ }^{4}$. Nossos dados revelam o oposto. Entre os funcionários do HC, apesar da prevalência ao longo da vida ter sido menor que a tradicionalmente descrita, a maior parte dos casos de cefaléia era de migrânea. Se considerarmos os casos que preencheram os critérios, com duração da dor menor que 4 horas, essa prevalência sobe para 44,7\% (Tabela 2). Questiona-se atualmente a arbitrariedade em se considerar como migranosos apenas aqueles cujas dores duram mais de 4 horas ${ }^{15}$. Por considerarmos que os demais fatores (intensidade, localização e caráter da dor, fenômenos acompanhantes) são tão ou mais importantes que a duração da dor na determinação da diminuição das capacidades, optamos por avaliar ambos os grupos conjuntamente.

Entre os funcionários do HC, 20,8\% apresentavam outros tipos de cefaléia que não a migrânea, dentre os quais a cefaléia do tipo tensional episódico. Mesmo considerando estudos de prevalência em profissionais de saúde, em que se encontraram valores maiores, nossos dados revelam a alta prevalência da migrânea dentre os trabalhadores do $\mathrm{HC}^{16}$. Esse fato também se verifica quando consideramos as prevalências anual e mensal. É interessante o fato de que $97 \%$ dos funcionários que preenchem critérios para migrânea ao longo da vida, tiveram dor no último ano e $89 \%$ no último 


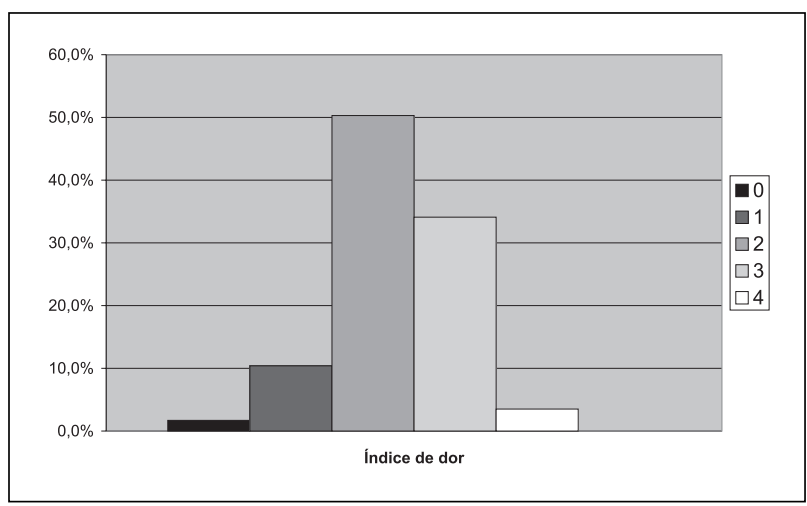

Fig 2. Percentual de funcionários com migrânea, de acordo com a categoria do índice de dor.

mês. Uma explicação possível é que nossos funcionários, além de expostos ao estresse inerente do trabalho que exercem, sofram os reveses de um cotidiano particularmente extenuante, que reúne baixos salários, sobrecarga de trabalho secundária ao acúmulo de empregos e outros. É comum auxiliares de enfermagem que se submetem a pouquíssimas horas de sono diariamente, pelo excesso de trabalho. Uma vez que alguns estudos sugerem maior prevalência de distúrbios ansiosos (ansiedade generalizada, fobias e transtorno do pânico) em migranosos ${ }^{17-19}$, é possível que o estresse descrito acarrete aumento dos escores de ansiedade nos funcionários da saúde, com repercussão na prevalência da cefaléia. Estudos que visam aprofundar tal correlação estão sendo conduzidos por nosso grupo.

Em relação à frequência de crises (Fig 1), nossos dados assemelham-se aos de Henry et al. ${ }^{16}$ que, em estudo nacional na França, encontraram a seguinte distribuição de frequência de crises: menos de 1 por mês, 17\%; 1/mês, 32\%; 2 a 4 por mês, $40 \%$; mais de 1 por semana, $10 \%$.

Quanto à intensidade da dor (Fig 2), estudos consideraram intensa ou muito intensa em 58 a $85 \%$ dos pacientes ${ }^{16,20}$. Cerca de $88 \%$ dos funcionários a referiram como intensa e mais de $50 \%$ relataram que, além de intensa, era pelo menos moderadamente limitante. Mostram ainda que a maioria dos funcionários pesquisados apresenta crises frequentes e intensas.

O impacto na qualidade de vida, bem como a diminuição das capacidades laborativa e de lazer, provocados pela migrânea, são fatores bem conhecidos e amplamente descritos na literatura. Em estudo de prevalência nos EUA realizado por Lipton e et al. ${ }^{7}$ foi avaliado o grau de diminuição das capacidades provocado pela migrânea: nenhum, $12 \%$; leve ou moderado, 51,3\%; intenso/vai para a cama, $35,5 \%$; não sabem, $1 \%$. Portanto, $86,8 \%$ dos pacientes pesquisados tinham diminuição das capacidades devido à migrânea. Em nosso estudo, a aferição pelo índice de dor crônica demonstrou que os funcionários do HC sentem-se prejudicados pela enxaqueca, no tocante a sua qualidade de vida. Verificamos que $35,1 \%$ deles evitam dirigir quando com dor, $59,9 \%$ já cancelaram atividades com a família, $81,2 \%$ cancelaram atividades de lazer e $82 \%$ ou têm que se deitar independentemente do que estão fazendo ou não podem mas gostariam (Tabela 3 ).

Classicamente, ainda que a migrânea seja condição crônica, entre os ataques o paciente tem sido considerado como de excelente saúde. Estudo recente mostrou que migranosos têm significativas limitações na qualidade de vida em relação a população saudável ${ }^{20}$. Mesmo quando comparados a outras condições crônicas, como artrose, depressão e diabetes, os migranosos obtiveram os piores escores, juntamente com os portadores de depressão ${ }^{19}$. Essa limitação que extrapola os limites da crise de dor também fica evidente em nossos dados (Tabela 4). Mais de $40 \%$ dos funcionários têm medo de ter outra crise e esse medo é suficiente para que pensem sempre na dor. Parcela não desprezível relatou impacto em seu relacionamento com a família, amigos ou atividade sexual. Foi demonstrado que mais de $75 \%$ dos migranosos evitam supostos fatores desencadeantes. Muitos têm 
afetada sua decisão acerca de empregos, evitam situações sociais ou festas que possam desencadear crises, o que indica que a migrânea diminui significativamente a qualidade de vida não somente durante os ataques, como também nos períodos intercríticos, quando ansiedade, medo e incerteza contribuem para uma gradual retirada da maioria dos contatos sociais ${ }^{21}$.

Nossos dados sugerem que a migrânea representa problema de saúde pública entre os funcionários do HC. Acarreta importante impacto na qualidade de vida deles, o que permite inferir haver diminuição de sua capacidade laborativa, com prejuízo econômico considerável. Cremos que este problema mereça, portanto, atenção especial, através de melhor diagnóstico e tratamento e possível adoção de medidas não farmacológicas (sala de repouso, atividades de relaxamento) para minimizá-lo.

\section{BIBLIOGRAFIA}

1. Olesen J. Discussion summary. In Olesen J. Headache classification and epidemiology. New York: Raven Press, 1994:227-228.

2. Rasmussen BK. Epidemiology of headache in Europe. In Olesen J Headache classification and epidemiology. New York: Raven Press, 1994:231-237.

3. Barea LM, Tannhauser M, Rotta NT. An epidemiological study of headache among children and adolescents of southern Brazil. Cephalalgia 1996;16:545-549.

4. Rasmussen BK. Epidemiology of headache. Cephalalgia 1995;5:45-68.

5. Bigal ME, Bordini CA, Speciali JG. Etiology and distribution of headaches in two brazilian primary care units. Headache (in press).

6. Silberstein SD, Lipton RB. Headache epidemiology: emphasis on migraine. Neurol Clin 1996;14:421-434.

7. Lipton RB, Silberstein MD, Stewart WF. An update on the epidemiology of migraine. Headache 1994;34:319-328.

8. The Migraine Disability and Optimizing Care (MIDAS). A poster presentation. New York: Zeneca Pharmaceuticals, 1997.

9. Lipton RB, Stewart WF. Prevalence and impact of migraine. Neurol Clin. 1997;15:1-13.

10. Matthew N. Chronic tension-type headache: diagnosis, clinical characteristics, and health impact. In Olesen J, Schoenen J. Tension-type headache: classification, mechanisms and treatment, New York: Raven Press, 1993;15-25.

11. Michel MD, Dartigues JF, Lindouisi MD. Loss of productivity and quality of life in migraine sufferers among French workers: results from the GAZEL cohort. Headache 1997;37:71-78.

12. Headache Classification Committee of the International Headache Society. Classification and diagnostic criteria for headache disorders cranial neuralgias and facial pain. Cephalalgia 1988;8(Suppl 7):1-96.

13. Von Korff M, Ormel J, Keefe F, et al. Grading the severity of chronic pain. Pain 1992;50:133-149.

14. Sanvito WL, Monzillo PH, Peres MF, et al.. The epidemiology of migraine in medical students. Headache 1996;36:316-319.

15. Sakai F, Higarachi H. Prevalence of migraine in Japan: a nationwide survey. Cephalalgia 1997;17:15-22.

16. Henry P, Michel P, Brochet P, Dartigues JF, Tison S, Salamon R. GRIM, a nationwide survey of migraine in France : prevalence and clinical features in adults. Cephalalgia 1992;12:229-237.

17. Stewart WS, Linet MS, Celentano DD. Migraine headaches and panic attacks. Pshychosom Med 1989;51:559-561.

18. Merikangas KR, Stevens DE, Angst J. Headache and personality: results of a community sample of young adults. J Psychiat Res 1993;27:187-196.

19. Breslau N, Andreski P. Migraine and psychiatric comorbity. Headache (In press).

20. Stang PE, Osterhaus J. Impact of migraine in the United States: data from the National Health Interview survey. Headache 1993;33:29-35.

21. Edmeads E, Findlay H. Impact of migraine and tension-type headache on life-style, consulting behavior and medication use: a Canadian population survey. Can J Neurol Sci 1993;20:131-137. 